\title{
The Potential of Targeting DNA Repair Deficiency in Acute Myeloid Leukemia
}

\author{
Clare M. Crean, Ken I. Mills*, Kienan I. Savage \\ Centre for Cancer Research and Cell Biology (CCRCB), Queen's University Belfast, Belfast, UK \\ Email: *k.mills@qub.ac.uk
}

How to cite this paper: Crean, C.M., Mills, K.I. and Savage, K.I. (2017) The Potential of Targeting DNA Repair Deficiency in Acute Myeloid Leukemia. Journal of Cancer Therapy, 8, 691-698. https://doi.org/10.4236/jct.2017.88060

Received: April 28, 2017

Accepted: July 25, 2017

Published: July 28, 2017

Copyright $\odot 2017$ by authors and Scientific Research Publishing Inc. This work is licensed under the Creative Commons Attribution International License (CC BY 4.0).

http://creativecommons.org/licenses/by/4.0/

\begin{abstract}
Acute myeloid leukemia (AML) is a clonal heterogeneous disease of the myeloid white blood cells. It is characterised by an accumulation of immature blast cells and a number of chromosomal and genetic mutations have been identified. In both de novo and therapy-related AML, defective DNA repair mechanisms are responsible for some of these genetic abnormalities. Targeting the DNA repair mechanism has been shown to be successful against certain forms of solid tumors and may represent a novel therapeutic approach for AML.
\end{abstract}

\section{Keywords}

DNA Repair, Acute Myeloid Leukemia

\section{Introduction}

Acute Myeloid Leukemia (AML) is a progressive disease of the myeloid blood line of hematopoietic cells [1]. A rapid growth of undifferentiated white blood cells is seen in AML, which causes a reduction in the number of mature and functioning red blood cells, white blood cells and platelets. AML is most commonly seen in people over the age of 65 but it can be seen in younger patients [2]. Although AML is caused by cytogenetic and genetic mutations [3], there are some rare inherited conditions, such as Fanconi Anemia, Bloom Syndrome and Downs Syndrome, which confer an increasing risk of developing a hematological malignancy [4].

Chromosomal translocations are a regular occurrence in AML. The prognosis of AML with chromosomal translocations is governed by the translocated genes involved [5]. The most common breakpoints happen in genes with highly important cellular functions such as the PML (ProMyelocytic Leukemia), RARA (Retinoic Acid Receptor alpha), TEL (also known at ETV6 (ETS family tran- 
scription factor variant 6)), MLL (Mixed Lineage Leukemia), ETO (Eight Twenty-Two, also known as RUNX1T1 (RUNX1 Translocation Partner 1)), BCR (Breakpoint Cluster Region), HOXA9 (Homeobox A9) and ABL1 (ABL ProtoOncogene 1) [5]. The fusion proteins (such as PML-RARA), created through numerous chromosomal translocations, block differentiation, which leads to abnormal blood cell phenotypes. Ineffective or incomplete DNA repair mechanisms are responsible for the formation of chromosomal translocations. Targeting the DNA repair pathway as a form of treatment is relatively new, and although it has been shown to be successful against certain forms of solid tumors, it has been used infrequently in leukemia [6].

\section{Therapy-Related AML}

The occurrence of AML can be de novo; however, a number of AML cases are therapy-related [7]. As the overall-survival rate of many cancers is increasing, so too is the number of living people who have received cytotoxic treatments. These people are at risk of developing secondary neoplasms, particularly haematological neoplasms. As a result of this, a rise in the frequency of patients presenting with therapy-related AML (t-AML) can be seen [7]. T-AML is categorised as any AML occurring in patients which have previously received cytotoxic drug treatments or radiotherapy which targeted active bone marrow sites [8]. These agents are capable of heavily mutating DNA and causing gross chromosomal rearrangements. Additionally DNA-repair pathways are often disrupted which leads to a build-up in cells with abnormally repaired / mutated DNA [9] [10].

\section{DNA Repair Pathways}

DNA repair mechanisms play an important role in the maintenance of healthy cells. Lack of sufficient repair mechanisms causes DNA damage which can lead to apoptosis, cell cycle arrest or even cancer [11]. DNA repair genes do not only confer a negative effect by loss of function mutations. Overexpression of DNA repair genes encourages cells to try and repair severe damage instead of undergoing apoptosis. This allows damaged cells to become resistant to chemotherapy and even targeted therapies [12]. As a result of sub-par DNA repairing, disease progression worsens due to the build-up of genomic mistakes. Moreover, increased expression levels of BRCA1, RAD51 and CHK2, among other DDR genes, are associated with a poor prognosis [12] [13]. Thus there is a growing of evidence to show the role played by DNA repair pathways in cancer prevention. Identifying differences between cancerous cells and non-cancerous cells is a key element in the production of targeted therapies. There are at least 6 with three major DNA repair pathways in human cells, each playing a different role in DNA maintenance (Figure 1): Base excision repair (BER); nucleotide excision repair (NER); mismatch repair (MMR); homologous recombination; Fanconi anemia/BRCA pathway (HR); non-homologous end-joining (NHEJ) and translesion DNA synthesis (TLS) [11]. Due to the multitude of DNA repair mechanisms, a defect in one pathway does not necessarily lead to cell death. In fact, a 
defect often leads to a damaged cell that will continue to grow and multiply with this defect creating a mass of cells imperfectly replicated. Double stranded breaks present serious problems for cells as they can cause major changes in sequence and even chromosome rearrangements (Figure 2). There are two major mechanisms for repairing double stranded breaks-homologous repair and nonhomologous end joining [14].

\subsection{Homologous Recombination}

Homologous recombination (HR) is the more complex, but also the most accurate, of the two methods. HR is initiated by sensing of the DSB by at the Ataxia Telangiectasia Mutated (ATM) Kinase which promotes the 5' - 3' resection of the broken strands, through recruitment and activation of the Mre11:Rad50:Nbs1 (MRN) complex (Mre11 harbours both exo- and endo-nuclease activity), resulting in a pair of single stranded 3 ' overhangs which recruit proteins to aid in the repair process (15). RPA is the first protein to bind to these 3 ' ends helping to stabilise and protect these single stranded DNA regions. The recombinase pro-

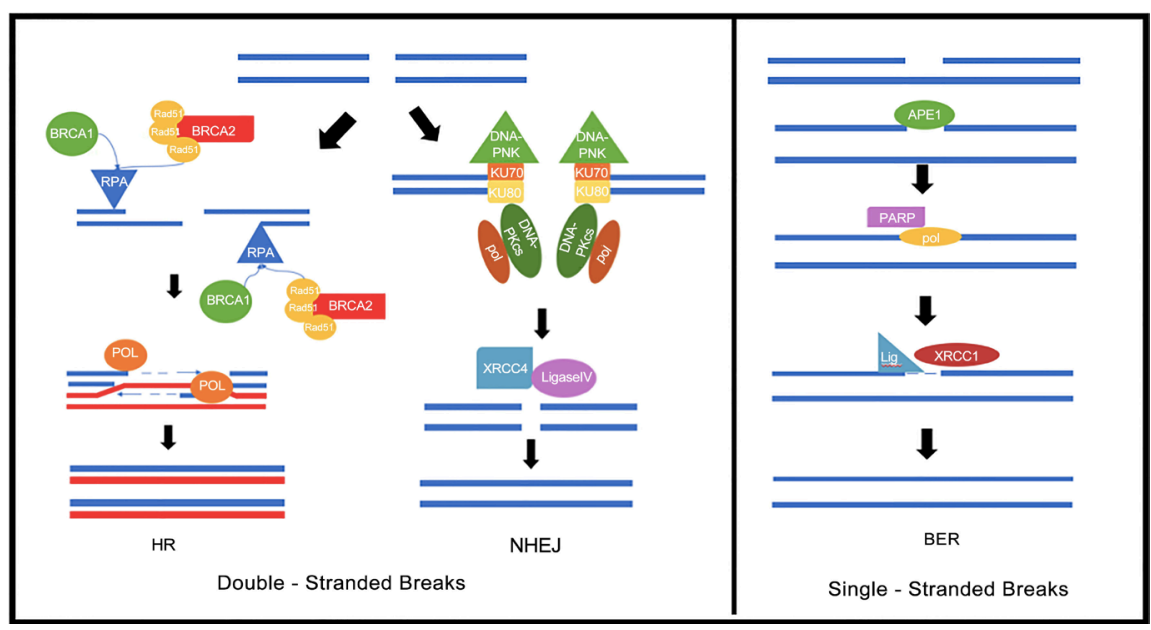

Figure 1. Three commonly used pathways in the repair of both single and double strand breaks.
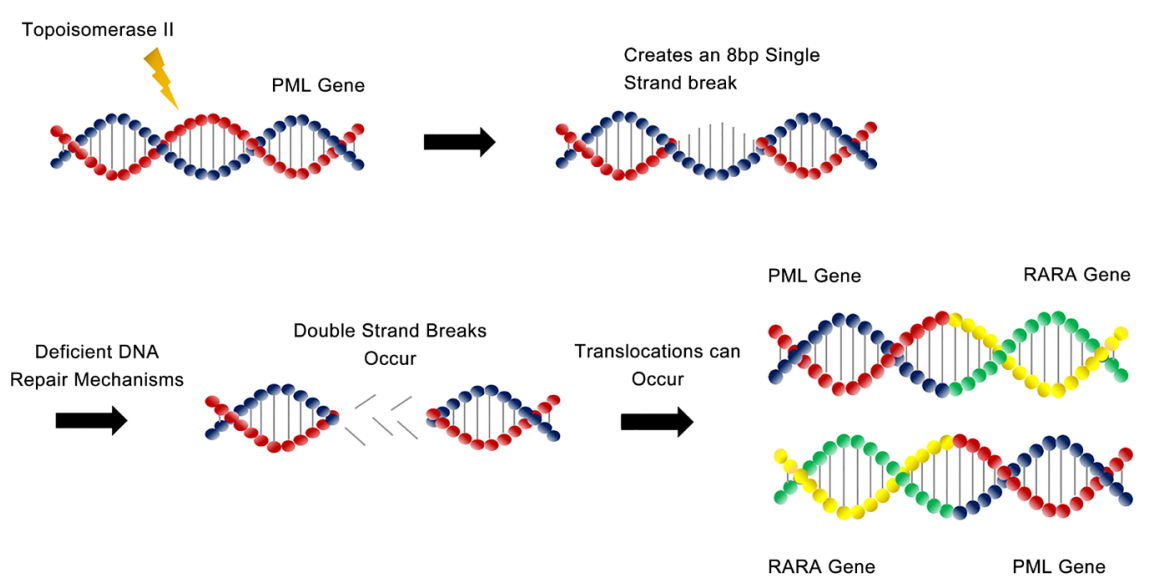

Figure 2. Schematic showing how topoisomerase has the potential to lead to chromosomal translocation via inefficient DNA repair. 
tein Rad51 then replaces RPA, a process driven by several mediating proteinssuch as the BRCA1 and BRAC2. Rad51 stimulates repair by catalysing the invasion of the sister strand and aligning the single stranded 3 ' overhang with its complementary region within the sister chromatid. This results in the formation of two replication fork like structures (known as a Holiday Junction), allowing the resected DNA to be replaced by the DNA replication machinery using the undamaged sister chromatid as a template [15] [16]. Although, the most accurate DSB repair pathway, as it relies on the presence of an intact sister chromatid, HR can only occur during S or G2 phases of the cell cycle. The correct balance of Rad51 and RPA proteins are crucial for efficient and successful HR mediated repair. Indeed, Rad51 overexpression has been observed in many cancer types including in breast cancer and in several sub-types of leukemia [16] [17].

As the HR repair pathway is so important in cell survival and correct replication, it has become a therapeutic target in cancer cells which already contain a DNA repair defects. Molecules which inhibit HR activity, such as Mirin, are in the early phases of development [18]. Mirin inhibits the nuclease activity of Mre11 within the MRN complex, thereby inhibiting the initiation of HR. These small molecule inhibitors, and others similar (such as RAD51 inhibitors), are still in early phase trials. These molecules target HR through various avenues [18]. Targeting CABL and HSP90 are also being investigated in HRR deficient cells [16].

\subsection{Non-Homologous End Joining}

Non-homologous end joining is a much quicker and simpler mechanism for DSB repair. During NHEJ, the two DNA strands are re-joined even if they do not have sequence homology. Interestingly, NHEJ activity has been shown to be increased in chronic myeloid leukemia [19]. However, defects are seen often in lymphoid malignancies [20] [21]. Over activity of NHEJ is seen in many cancers, likely due to the high level of DNA damage found in cancers and/or through compensation due to HR defects in these cells. As NHEJ and HR are the only DSB repair pathways, loss of both pathways would likely to result in synthetic lethality in cancer cells. As discussed earlier, there are already a number of HR targeted therapies in early phase trials, which may be of benefit in tumours with NHEJ defects. However, the more common defect observed in cancer are HR defects, which result in NHEJ, the error prone DSB repair pathway becoming the predominant repair pathway in these cells (and thus leading to a more mutagenic (phenotype). Thus it is likely that NHEJ inhibitors may be more promising as cancer therapeutics. The subunit of DNA-PKCs is a promising target for inhibition as it plays an important role in NHEJ and is shown to confer radio-resistance in a number cancer types when overexpressed. Although Wortmannin was the first drug synthesised to target NHEJ, many more specific agents have since been developed, with some entering phase I and phase II trials [14] [21]. 


\section{DNA Repair Pathway Inhibitors}

\subsection{PARP 1 Inhibitors}

The Poly (ADP-ribose) polymerase, PARP, gene encodes a 1014 AA protein that is involved in many cellular processes, including cell cycle regulation and apoptosis [21] [22]. One of PARP most important functions, however, is in the DNA damage response. PARP is active in the repair of both single stranded and double stranded DNA breaks with PARPs playing a major role in the BER (responsible for the repair of single strand breaks and modified/damaged bases) and the NHEJ pathways [21].

The PARP family of enzymes catalyse the movement of ADP-ribose from the $\mathrm{NAD}^{+}$(nicotinamide adenine dinucleotide) subunit onto target proteins. PARP1 is recognised as the most important of the PARP family proteins [23] [24]. PARP1 generates a long strand of poly (ADP-ribose) which can covalently attach to target proteins assisting DNA repair as well as other cellular processes [25]. When PARP1 is inhibited, BER loses its ability to repair SSBs. These breaks subsequently evolve into DSBs during replication. This results in an overwhelming amount of damage for the cells to deal with particularly in the background of an already depleted DSB repair system [16]. Apoptosis generally follows soon after as the genetic mutations reach a lethal level. The idea of synthetic lethality was introduced to target cells that already contain an intrinsic repair pathway defect. While one defect is not generally detrimental to a cells survival, the synthetic introduction of another pathway defect can be enough to push a cell into such disarray that the only option it faces is apoptosis [23].

As homologous recombination repair deficient cells are so dependent on the BER pathway, in which PARP1 plays a key role, targeting PARP1, is seen as an effective method of targeting these cells [21] [23]. Additionally, some biomarkers now highlight any defects in DNA repair mechanisms. Indeed, the levels of PARP1 itself may act as a biomarker with over-active PARP1 activity suggest that the tumour or leukemia may be responsive to PARP inhibition. Mutated BRCA is the most recognised and reliable biomarker for DNA repair deficiency but many others, including CDK12 and FA proteins, are also used in repair deficiency analysis [26].

DNA repair inhibitors have the unique ability to target cancerous cells while leaving normal cells unharmed. Despite the clear logic behind the use of PARP inhibitors (PARPi) in DNA repair deficient tumours, the development of early PARPi were not as successful in the clinic. The initial PARP1 inhibitor created, Iniparib, was unsuccessful in a phase III trial when used as a treatment for triple-negative breast cancer [26]. It also proved to be ineffective in a phase II non-small lung cancer trial. Indeed, the selectivity of Iniparib was low, as was its ability to efficiently inhibit PARP1. PARPi designed to confer a stronger affinity for PARP1 and a greater rate of inhibition. Despite early clinical trial doubts, one such inhibitor Olaparib has had very positive results in the treatment of BRCA-deficient ovarian cancer and in unfavorable AML [27]. More recently, the 
use of Olaparib has been approved as a treatment option for prostate cancer with either a BRCA or ATM mutation [26]. A further nine PARP1 inhibitors are currently in different stages of clinical trials, amongst which Rucaparib and Talazoparib have shown promising results [26].

\subsection{Other DNA Repair Pathway Inhibitors}

Targeting DNA repair pathways is relatively new in the field of AML yet interesting results seen in other tumor types suggest a promising future in this area of AML therapeutics.

Although PARP inhibitors have been heavily focused on in recently, there are many aspects of DNA repair system that may be targeted for future cancer treatments.

As previously stated, the overexpression of repair pathway components such as DNA-PKCs and BRCA-1 can also confer a negative impact [13]. Recent studies have identified an up regulation of similar elements in chemotherapy resistant AML cell lines. Indeed Nishida et al showed that DNA-PKCs and DNA Ligase IV are up-regulated in K562 and HL60 cells which are resistant to daunorubicin; whilst knocking down DNA-PKC's in these cells induces sensitivity to daunorubicin [28].

DNA repair inhibitors need not be used as single agents. By combining them with therapies which target other mutations they insure the cell has no back-up pathway to depend on. For example, PARPi can be used in conjunction with Temozolomide to inhibit the use of the BER pathway which is often utilised by Temolozolomide-resistant cells [27].

\section{Conclusion}

Although AML is clearly a disease of immense heterogeneity particularly with respect to the distinct genetic mutations found in this disease. However, it is through targeting these disparities directly that effective treatments are likely to be found. The targeting of DNA repair deficiency is an area of unmet clinical need in AML. Few people have derived links between genetic mutations in AML and possible targets for repair pathway inhibitors. Based on promising results in other cancer types, it is likely to be an emerging area of research in AML as well as other hematological malignancies.

\section{Acknowledgements}

CMC was funded by a grant (R2301CNR) from Leukaemia Lymphoma NI to KIM and KS.

\section{References}

[1] Link, D.C. (2012) Molecular Genetics of AML. Best Practice \& Research Clinical Haematology, 25, 409-414. https://doi.org/10.1016/j.beha.2012.10.002

[2] Shlush, L.I. and Mitchell, A. (2015) AML Evolution from Preleukemia to Leukemia and Relapse. Best Practice \& Research Clinical Haematology, 28, 81-89. 
https://doi.org/10.1016/j.beha.2015.10.004

[3] Papaemmanuil, E., Gerstung, M., Bullinger, L., Gaidzik, V.I., Paschka, P., Roberts, N.D., et al. (2016) Genomic Classification and Prognosis in Acute Myeloid Leukemia. The New England Journal of Medicine, 374, 2209-2221.

https://doi.org/10.1056/NEJMoa1516192

[4] Esposito, M.T. and So, C.W. (2014) DNA Damage Accumulation and Repair Defects in Acute Myeloid Leukemia: Implications for Pathogenesis, Disease Progression, and Chemotherapy Resistance. Chromosoma, 123, 545-561.

https://doi.org/10.1007/s00412-014-0482-9

[5] Grimwade, D., Hills, R.K., Moorman, A.V., Walker, H., Chatters, S., Goldstone, A.H., et al. (2010) Refinement of Cytogenetic Classification in Acute Myeloid Leukemia: Determination of Prognostic Significance of Rare Recurring Chromosomal Abnormalities among 5876 Younger Adult Patients Treated in the United Kingdom Medical Research Council Trials. Blood, 116, 354-365.

https://doi.org/10.1182/blood-2009-11-254441

[6] Gavande, N.S., VanderVere-Carozza, P.S., Hinshaw, H.D., Jalal, S.I., Sears, C.R., Pawelczak, K.S., et al. (2016) DNA Repair Targeted Therapy: The Past or Future of Cancer Treatment? Pharmacology \& Therapeutics, 160, 65-83.

[7] Casorelli, I., Bossa, C. and Bignami, M. (2012) DNA Damage and Repair in Human Cancer: Molecular Mechanisms and Contribution to Therapy-Related Leukemias. International Journal of Environmental Research and Public Health, 9, 2636-2657. https://doi.org/10.3390/ijerph9082636

[8] Hasserjian, R.P. (2013) Acute Myeloid Leukemia: Advances in Diagnosis and Classification. International Journal of Laboratory Hematology, 35, 358-366. https://doi.org/10.1111/ijlh.12081

[9] Zhang, Y. and Rowley, J.D. (2006) Chromatin Structural Elements and Chromosomal Translocations in Leukemia. DNA Repair (Amst), 5, 1282-1297.

[10] Churpek, J.E. and Larson, R.A. (2013) The Evolving Challenge of Therapy-Related Myeloid Neoplasms. Best Practice \& Research Clinical Haematology, 26, 309-317. https://doi.org/10.1016/j.beha.2013.09.001

[11] D'Andrea, A.D. (2010) Targeting DNA Repair Pathways in AML. Best Practice \& Research Clinical Haematology, 23, 469-473.

[12] Rollinson, S., Smith, A.G., Allan, J.M., Adamson, P.J., Scott, K., Skibola, C.F., et al. (2007) RAD51 Homologous Recombination Repair Gene Haplotypes and Risk of Acute Myeloid Leukaemia. Leukemia Research, 31, 169-174.

[13] Esposito, M.T., Zhao, L., Fung, T.K., Rane, J.K., Wilson, A., Martin, N., et al. (2015) Synthetic Lethal Targeting of Oncogenic Transcription Factors in Acute Leukemia by PARP Inhibitors. Nature Medicine, 21, 1481-1490. https://doi.org/10.1038/nm.3993

[14] Kelley, M.R., Logsdon, D. and Fishel, M.L. (2014) Targeting DNA Repair Pathways for Cancer Treatment: What's New? Future Oncology, 10, 1215-1237. https://doi.org/10.2217/fon.14.60

[15] Worrillow, L.J. and Allan, J.M. (2006) Deregulation of Homologous Recombination DNA Repair in Alkylating Agent-Treated Stem Cell Clones: A Possible Role in the Aetiology of Chemotherapy-Induced Leukaemia. Oncogene, 25, 1709-1720. https://doi.org/10.1038/sj.onc.1209208

[16] Daley, J.M., Gaines, W.A., Kwon, Y. and Sung, P. (2014) Regulation of DNA Pairing in Homologous Recombination. Cold Spring Harbor Perspectives in Biology, 6, a017954. https://doi.org/10.1101/cshperspect.a017954 
[17] Jacoby, M.A., De Jesus Pizarro, R.E., Shao, J., Koboldt, D.C., Fulton, R.S., Zhou, G., et al. (2014) The DNA Double-Strand Break Response Is Abnormal in Myeloblasts from Patients with Therapy-Related Acute Myeloid Leukemia. Leukemia, 28, 1242 1251. https://doi.org/10.1038/leu.2013.368

[18] Lai, T.H., Ewald, B., Zecevic, A., Liu, C., Sulda, M., Papaioannou, D., et al. (2016) HDAC Inhibition Induces MicroRNA-182, Which Targets RAD51 and Impairs HR Repair to Sensitize Cells to Sapacitabine in Acute Myelogenous Leukemia. Clinical Cancer Research, 22, 3537-3549. https://doi.org/10.1158/1078-0432.CCR-15-1063

[19] Chakraborty, S., Stark, J.M., Sun, C.L., Modi, H., Chen, W., O’Connor, T.R., et al. (2012) Chronic Myelogenous Leukemia Stem and Progenitor Cells Demonstrate Chromosomal Instability Related to Repeated Breakage-Fusion-Bridge Cycles Mediated by Increased Nonhomologous End Joining. Blood, 119, 6187-6197. https://doi.org/10.1182/blood-2011-05-352252

[20] Ralser, M., Albrecht, M., Nonhoff, U., Lengauer, T., Lehrach, H. and Krobitsch, S. (2005) An Integrative Approach to Gain Insights into the Cellular Function of $\mathrm{Hu}$ man Ataxin-2. Journal of Molecular Biology, 346, 203-214.

[21] Robert, C., Nagaria, PK., Pawar, N., Adewuyi, A., Gojo, I., Meyers, D.J., et al. (2016) Histone Deacetylase Inhibitors Decrease NHEJ Both by Acetylation of Repair Factors and Trapping of PARP1 at DNA Double-Strand Breaks in Chromatin. Leukemia Research, 45, 14-23.

[22] Langelier, M.F. and Pascal, J.M. (2013) PARP-1 Mechanism for Coupling DNA Damage Detection to Poly(ADP-Ribose) Synthesis. Current Opinion in Structural Biology, 23, 134-143.

[23] Zhao, L. and So, E.C.W. (2016) PARP-Inhibitor-Induced Synthetic Lethality for Acute Myeloid Leukemia Treatment. Experimental Hematology, 44, 902-907.

[24] Wang, L., Cai, W., Zhang, W., Chen, X., Dong, W., Tang, D., et al. (2015) Inhibition of Poly(ADP-Ribose) Polymerase 1 Protects against Acute Myeloid Leukemia by Suppressing the Myeloproliferative Leukemia Virus Oncogene. Oncotarget, 6, 27490-27504. https://doi.org/10.18632/oncotarget.4748

[25] Sukhanova, M., Khodyreva, S. and Lavrik, O. (2010) Poly(ADP-Ribose) Polymerase 1 Regulates Activity of DNA Polymerase Beta in Long Patch Base Excision Repair. Mutation Research, 685, 80-89.

[26] Wang, Y.Q., Wang, P.Y., Wang, Y.T., Yang, G.F., Zhang, A. and Miao, Z.H. (2016) An Update on Poly(ADP-Ribose)Polymerase-1 (PARP-1) Inhibitors: Opportunities and Challenges in Cancer Therapy. Journal of Medicinal Chemistry, 59, 9575-9598. https://doi.org/10.1021/acs.jmedchem.6b00055

[27] Muvarak, N.E., Chowdhury, K., Xia, L., Robert, C., Choi, E.Y., Cai, Y., et al. (2016) Enhancing the Cytotoxic Effects of PARP Inhibitors with DNA Demethylating Agents-A Potential Therapy for Cancer. Cancer Cell, 30, 637-650.

[28] Nishida, Y., Mizutani, N., Inoue, M., Omori, Y., Tamiya-Koizumi, K., Takagi, A., et al. (2014) Phosphorylated Sp1 Is the Regulator of DNA-PKcs and DNA Ligase IV Transcription of Daunorubicin-Resistant Leukemia Cell Lines. Biochimica et Biophysica Acta, 1839, 265-274. 
Submit or recommend next manuscript to SCIRP and we will provide best service for you:

Accepting pre-submission inquiries through Email, Facebook, LinkedIn, Twitter, etc. A wide selection of journals (inclusive of 9 subjects, more than 200 journals)

Providing 24-hour high-quality service

User-friendly online submission system

Fair and swift peer-review system

Efficient typesetting and proofreading procedure

Display of the result of downloads and visits, as well as the number of cited articles Maximum dissemination of your research work

Submit your manuscript at: http://papersubmission.scirp.org/

Or contact jet@scirp.org 